\title{
Brief report of the effects of seven human drugs studied on ants as models
}

\begin{abstract}
Since 2012, we used ants as models for examining the effects of products used by humans. Here, we summarize our seven last studies which concerned green clay, glucosamine, sildenafil, a homeopathic drug, as well as diclofenac, meloxicam and etoricoxib, three anti-inflammatory drugs. Green clay appeared to be an excellent product without adverse effects. In humans, it allows reducing gastric hyperacidity and should thus be used instead of aluminum hydroxide or calcium + magnesium carbonates which present adverse effects. Glucosamine was also safe. It only increased the ants' locomotion and this effect might explain, at least partly, its efficiency. Sildenafil has many adverse effects, the cause of which being probably a decrease of sensitive perception. Such an impact may result from the activity of sildenafil: it inhibits phosphodiesterases and increases amounts of cGMP and cAMP. The homeopathic drug Ignatia amara, used for decreasing stress symptoms, had no adverse effects and unexpectedly reduced the ants' state of stress. Even if its functioning stays unknown, this drug could help stressed persons. Diclofenac had several adverse effects and may lead to dependence; meloxicam had far less adverse effects and did not lead to dependence; etoricoxib had some adverse effects and did not lead to dependence. Obviously, meloxicam was the safest of the three anti-inflammatory drugs Experimentation on ants can thus inform practitioners and pharmacists on potential harmful effects of products before providing them to humans.
\end{abstract}

Keywords: diclofenac, etoricoxib, glucosamine, green clay, Ignatia amara, meloxicam, sildenafil
Volume 4 Issue 2 - 2019

\author{
Marie Claire Cammaerts \\ Independent researcher, retired from the Biology of Organisms \\ Department, University of Brussels, Belgium
}

Correspondence: Marie-Claire Cammaerts, Independent researcher, retired from the Biology of Organisms Department, 27 Square du Castel Fleuri, I I 70 Bruxelles, Belgium, Tel 322673 49 69, Email mccammaerts@gmail.com

Received: April 27, 2019 | Published: May 14, 2019
Abbreviation: ang.deg./cm, angular degrees per centimeter; $\mathrm{cm}$, centimeter; $\mathrm{mm}$, millimeter

\section{Introduction}

Examining effects of manmade electromagnetism on ants revealed that these insects could be used as biological models. ${ }^{1}$ Indeed, physiology and ethology are similar in most animals ${ }^{2}$ and research is generally firstly made on animals as models (e.g. fruit flies, cockroaches, bees, mice, monkeys) then on humans. ${ }^{3}$ Insects are often used, and ants could thus be used. ${ }^{4}$ They are eu-social, live in highly organized colonies, and detain many cognitive abilities. Their complex biology allows them to serve as biological models. We have largely worked on species of the genus Myrmica, and have a good knowledge of their ecology, eyes morphology, angle of vision, visual perception, recruitment strategies, navigation systems, conditioning, and ontogenesis of some of their social behaviors, ${ }^{5,6}$ as well as of their cognitive abilities and their limits. ${ }^{7,8}$ It is easy to detect the effects of products on these ants' locomotion, sinuosity of movement, orientation ability, audacity, tactile perception, brood caring, aggressiveness against nestmates, aggressiveness against aliens, cognition, escaping ability, conditioning capability and short term memory, adaptation to adverse effects, habituation to beneficial effects, and dependence on the drug consumption. The decrease of the effect of the drug after weaning can also be studied. This is why we have examined the effects of up to 39 products used by humans, giving precision on some effects observed in humans, as well as revealing other effects liable to exist in humans. All these works are published. They are also summarized in three papers, ${ }^{9-11}$ except the last seven ones which we summarize in the present paper. Here below, we very shortly recall our methods largely explained in previous works, we report the most important findings concerning the seven last studied products, and we present a few illustrations not published in the papers relative to these last studies.

\section{Material and methods}

\section{Maintenance of ants, feeding them with a drug}

The ants, collected from different sites, were maintained in laboratory in glass tubes half filled with water deposited in a tray $(34 \mathrm{~cm} \times 23 \mathrm{~cm} \times 4 \mathrm{~cm})$ which served as foraging areas. The ants were fed with an aqueous solution of sugar (30\%) provided ad libitum in a small glass tube plugged with cotton, and with pieces of Tenebrio molitor larvae (Linnaeus, 1758) delivered three times a week. Laboratory temperature was $18^{\circ} \mathrm{C}-22^{\circ} \mathrm{C}$, the relative humidity circa $80 \%$, the lighting $330 \mathrm{lux}$, the electromagnetic field $2-3 \mu \mathrm{W} / \mathrm{m}^{2}$. After having made all the control assessments, the sugar water was replaced by an aqueous sugared solution of the product to be examined. The concentration of the product was established on the basis of the amount of that product usually consumed by humans and on the fact that insects proportionally drink about ten times less than mammals.

\section{Products examined on ants}

Green clay is a natural product used in case of infections, skin irritations, ulcerations and digestive track problems. Until now, no adverse effect has been reported on its use, except that, during the first days of its consumption, it induces the elimination of waste elements, and that, after a few months of consumption, a recovering period is required (www.justebien.fr/bienfaits-argile-verte/). Glucosamine is a synthesized product use for treating arthritis. Its efficiency is nowadays debated, ${ }^{12}$ and even if generally estimated as being safe, ${ }^{13}$ some adverse effects have been reported. ${ }^{14}$ Sildenafil is a molecule which inhibits the type 5 phosphodiesterase, the level of cyclic GMP 
being thus maintained, and the smooth muscles staying relaxed. This allows keeping a man's erection. However, sildenafil somewhat acts on several other phosphodiesterases which are very important for any organism's physiology. It has thus some adverse effects, which are effectively mentioned in the instructions for use joined to this drug (Viagra ${ }^{\circledR}$ or Verventi ${ }^{\circledR}$ ) package. Homeopathy consists in treating patients with largely diluted toxic products. Scientific studies conclude that such a treatment is not at all efficient and may even be dangerous. ${ }^{15}$ However, sometimes, doubt is emitted as for a total inefficiency. ${ }^{16,17}$ Researchers experimenting on humans are confronted to the placebo effect. Therefore, experiments on biological models unable to present placebo effect is of interest.

Diclofenac as well as meloxicam are two anti-inflammatory drugs 'of the first generation'. Diclofenac is largely used and has been considered as safe during many years. However, a drastic decline of vultures occurred, due to their consumption of carcasses of domestic livestock treated with Diclofenac..$^{18}$ Danish researchers made a large meta-study ${ }^{19}$ and concluded that persons treated with diclofenac have a higher probability of suffering from cardiovascular problems than persons treated with paracetamol, ibuprofen or naproxen. Studies of the potential adverse effects of that drug on models having no inflammatory reaction (which causes in itself adverse effects) may thus be useful. Meloxicam_was proved to be less toxic for vultures, and many studies comparing diclofenac and meloxicam, essentially as for their impact on the digestive track, the liver and the kidneys, leaded to the conclusion that the later drug seemed safer. ${ }^{20}$ However, these studies did not examine the impact of the drug on, among others, the food consumption, activity, cognition, memory, social relationships, adaptation and dependence. It was thus useful to examine the effects of the two drugs on these physiological and ethological traits using 'blind' biological models. A second generation of anti-inflammatory drugs has been produced, the 'coxibs', which more selectively inhibit the COX-2 enzyme and induce therefore less gastrointestinal problems. ${ }^{21,22}$ They were thus preferred to the previous anti-inflammatory drugs. However, they appeared to cause high risks of cardiovascular events. ${ }^{22,23}$ Having studied the potential harmful impacts of diclofenac and meloxicam using ants as models, we of course studied in the same way those of a 'coxib', etoricoxib.

\section{Assessment of ants' traits}

The ants' sugar water and meat consumption as well as their general activity were assessed by counting during six days, six times per day, the ants present on the sugar water, on the $T$. molitor larvae, as well as those moving at any place in their environment, and establishing the daily means and the total mean for these three kinds of counts. Linear and angular speeds of freely moving ants were assessed without presenting them a stimulus, and their orientation towards an alarm signal was assessed while presenting them an isolated worker's head. Each time, 40 ants' trajectories were analyzed using specifically designed software. ${ }^{24}$ Each distribution of variable was characterized by its median and quartiles. The ants audacity was evaluated by counting, 10 times over $10 \mathrm{~min}$, the ants present on a cylindrical tower (height $=4 \mathrm{~cm}$; diameter $=1.5 \mathrm{~cm}$ ) and on its platform, and by establishing the mean and the extremes of the obtained values. The ants' tactile perception was evaluated through their locomotion on a rough substrate. When well perceiving the uncomfortable character of the substrate, the ants moved there very slowly and sinuously. The ants' brood caring behavior was assessed by removing larvae from the nest and counting the larvae among ten not re-entered in the course of 10 minutes. The ants' aggressiveness against nestmates as well as against aliens was examined in the course of dyadic encounters conducted in a cup (diameter $=2 \mathrm{~cm}$, height $=$ $1.6 \mathrm{~cm}$ ), the behavior of each ant being characterized by the numbers of times it did nothing (level 0 of aggressiveness), touched the other ant with its antennae (level 1), opened its mandibles (level 2), gripped and/or pulled the other ant (level 3), tried to sting or stung the other ant (level 4). The ants' aggressiveness was also assessed by 'a' which equaled the number of recorded aggressiveness levels $2+3+4$ divided by the number of levels $0+1$.

The ants' cognition was assessed by counting over 12 minutes those present in a small area in front of a twists and turns path, and those having crossed this difficult path. For approaching the effect of a substance on the ants' state of stress, we imprisoned 5 ants under a reversed polyacetate glass set on the foraging area, the rim of the bottom of which having a small notch allowing the ants escaping. The calmest were the ants and the more intact was their cognition, the more quickly they could escape. We assessed the ants' escaping ability by counting the ants still under the glass over 12 minutes, and by the number of escaped ants after 12 minutes among the initially enclosed ones. The ants' acquisition of operant visual conditioning, as well as their visual memory, was assessed by setting a visual cue on or near the ants' food, and by testing ants individually over days in a $\mathrm{Y}$-apparatus provided with the cue in one of its branches. Choosing the way with the cue was considered as giving the 'correct' choice. The percentage of correct responses obtained over time assessed the ants' conditioning acquisition, then after removal of the cue, the ants' memory.

Knowing at least one adverse effect of a product, the ants' adaptation to that effect could be assessed. Adaptation occurred when the ants no longer suffered from that adverse effect after several days of the product consumption. Leaning on at least one beneficial effect of a product, the ants' habituation to the product could be assessed. Habituation occurred when the beneficial effect of the product lowered in the course of its consumption. Dependence on a product was evaluated by transferring ants consuming that product into a tray $(15 \mathrm{~cm} \times 7 \mathrm{~cm} \times 5 \mathrm{~cm})$ provided with a tube containing sugar water, and a tube containing a sugared solution of the product. The ants drinking each liquid were counted 15 times over 15 minutes, and the values obtained compared to those expected if ants went randomly drinking the two liquids. The decrease, in the course of time, of an effect of a product after its consumption was stopped was assessed by replacing the sugared solution of the product by pure sugar water, and quantifying this effect over time.

Each time, the results were statistically analyzed using appropriate non-parametric tests.

\section{Results and discussion}

\section{Green clay}

Green clay did not impact the ants' food consumption, general activity, orientation ability, audacity, tactile perception, brood caring (Figure 1A), aggressiveness against nestmates and aliens, cognition, escaping capability, and conditioning acquisition. It somewhat ameliorated their memory. Ants developed no dependence on green clay consumption. After its consumption was stopped, the ants had to recover for about 8 hours and seemed then in perfect health. Humans could thus timely use green clay for ameliorating their health, and could use it in case of stomach hyperacidity instead of consuming other somewhat unsafe drugs. Read more in. ${ }^{25}$ 


\section{Glucosamine}

Glucosamine decreased the ants' food consumption, orientation ability and aggressiveness against aliens. It did not impact their tactile perception, brood caring behavior, aggressiveness against nestmates, cognition, escaping capability, conditioning acquisition and memory. Glucosamine increased the ants' activity, locomotion and audacity (Figure 1B). Ants did not adapt themselves to the effect of glucosamine on their locomotion and activity. They developed no dependence on that product consumption. After weaning, the effect of glucosamine slowly vanished in about 14 hours, decreasing in two successive steps, a first one, slowing down over time, and a second one, accelerating over time. Glucosamine is thus a safe product, simply somewhat increasing the individuals' activity and locomotion, and this impact may be the cause, or at least one important cause, of its efficiency in treating arthritis. Find details in. ${ }^{26}$

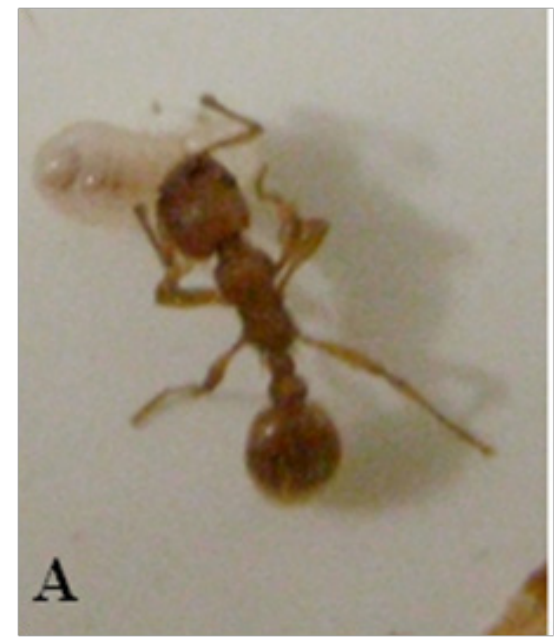

Figure IA An ant consuming green clay and taking care of a larva.

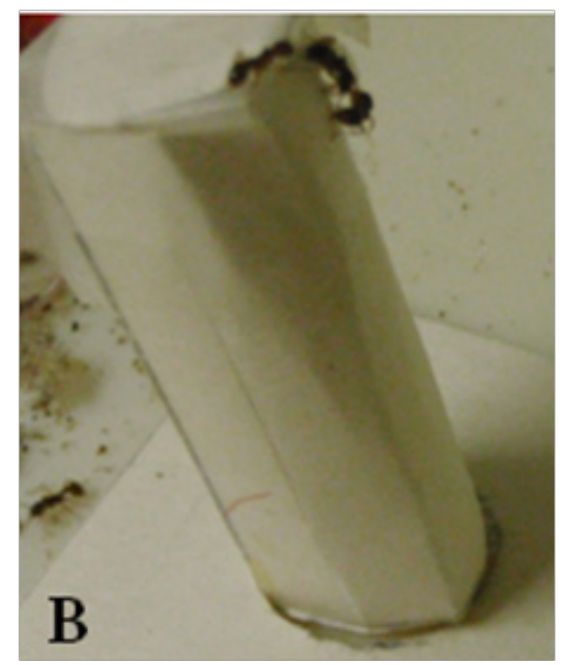

Figure IB Ants consuming glucosamine and climbing on a tower, a risky task.

\section{Sildenafil}

Sildenafil decreased the ants' meat consumption, locomotion (essentially near the nest entrance), orientation ability, audacity, tactile perception (Figure 1C\&1D), aggressiveness against aliens, and escaping capability. It did not impact the ants' sugar water consumption, general activity, brood caring, aggressiveness against nestmates, cognition and acquisition of conditioning. Sildenafil somewhat increased the ants' memory. The ants' visual as well as olfactory perception was significantly impacted by sildenafil consumption. Ants did not adapt themselves to the effect of sildenafil on their perception, on the contrary. They developed no dependence on that product consumption. After weaning, the effect of sildenafil slowly decreased in about 14 hours. Consequently, sildenafil impacted every ethological and physiological trait which required intact visual and olfactory perception. Sildenafil reduced thus such perceptions. This is due to the mode of action of the product: it inhibits a phosphodiesterase, and such enzymes allow the functioning of the sensory organs. Therefore, it is not safe to consume large amounts of sildenafil and to consume it during long time periods. Its use should be limited as much as possible, and restricted to absolute necessity. More information is available in. ${ }^{27}$

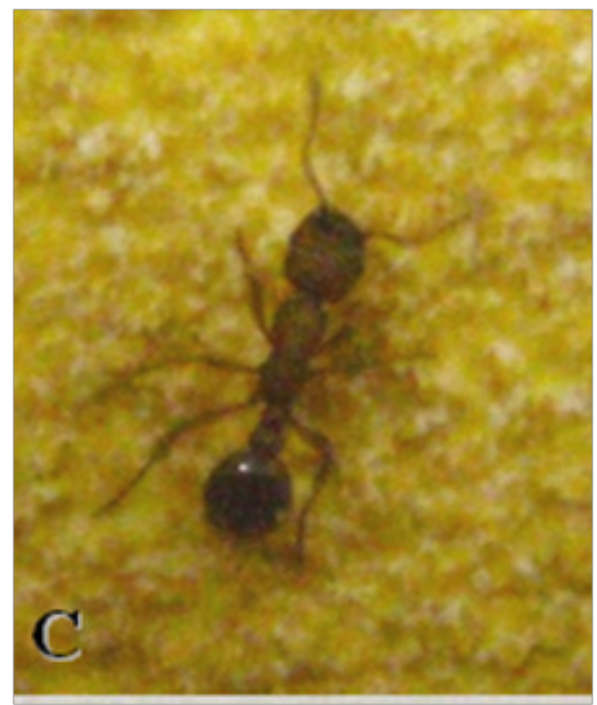

Figure IC an ant under normal diet having difficulties for walking on a rough substrate.

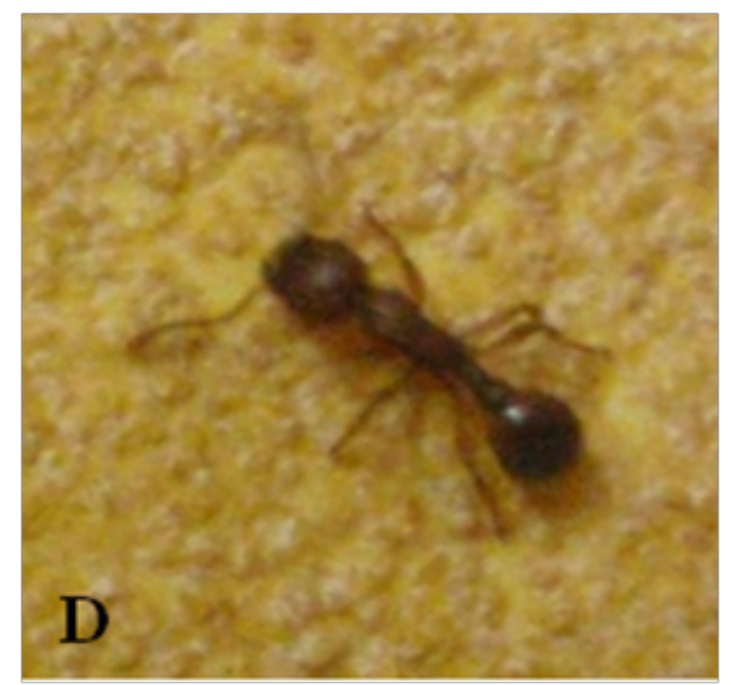

Figure ID An ant under sildefanil diet moving nearly as usual on a rough substrate, weakly perceiving its uncomfortable character. 


\section{Ignitia amara}

This homeopathic drug appeared to somewhat impact ants' physiological and ethological traits. It slightly affected their locomotion, orientation, audacity, tactile perception, brood caring, aggressiveness, cognition and memory, and the ants never adapted themselves to these effects. However, given to ants set in a stressing situation (they were maintained in presence of onion and had consequently ethological and ethological traits largely impacted), Ignatia amara unexpectedly appeared to significantly decrease the ants' state of stress and to restore their initially impacted traits. It ameliorated among others the ants' locomotion, orientation, audacity, tactile perception, brood caring, aggressiveness, escaping capability (Figure 1E) and cognition, and the ants never habituated themselves to these beneficial effects. The ants developed no dependence on the homeopathic drug consumption. Consequently, in some cases, i.e. for some health problems and using some adequate homeopathic drugs at low dilutions, homeopathy may be efficient and appreciated by patients. Practitioners should not refuse its use by patients, and should then go on following these consumers. Details are given in. ${ }^{28}$

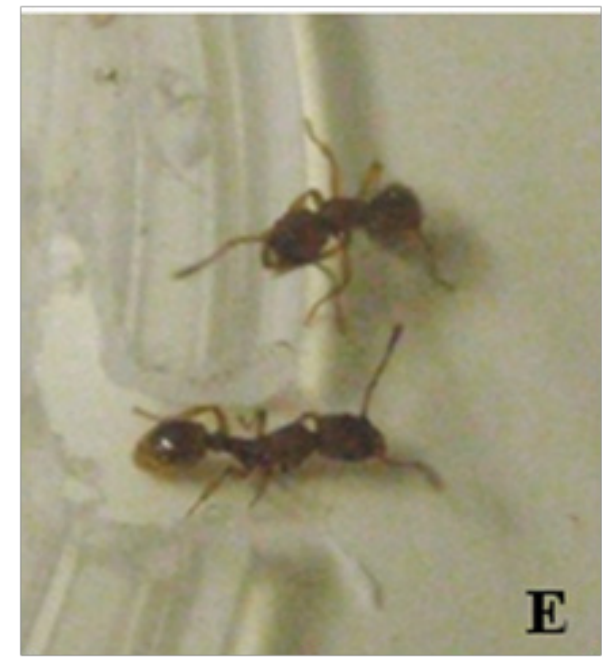

Figure IE An ant consuming the homeopathic drug Ignatia amara, having its stress, caused by onion odor, largely reduced and being again able to escape from the enclosure.

\section{Diclofenac}

This anti-inflammatory drug did not impact the ants' tactile perception, brood caring and aggressiveness. It increased their sinuosity of movement. Diclofenac significantly decreased the ants' food consumption, orientation ability, audacity, cognition (Figure $1 \mathrm{~F})$, escaping capability, conditioning acquisition and short term memory. The ants did not adapt themselves to these adverse effects, and developed some dependence on the drug consumption. After its consumption was stopped, the effect of diclofenac rapidly decreased in 6 hours. As many times observed, dependence on a product occurred when the effect of this product rapidly decreases after weaning. ${ }^{29}$ The anti-inflammatory drug diclofenac has thus adverse effects, and is efficient only during a short time period what leads to dependence. It should so be used very cautiously. The entire study is in..$^{30}$

\section{Meloxicam}

This anti-inflammatory drug did not affect the ants' locomotion, orientation ability, tactile perception, brood caring, conditioning acquisition and memory. It somewhat improved their social relationships (Figure 1G). Meloxicam only decreased the ants' food intake, activity, audacity, cognition and escaping capability. The ants did not adapt themselves to the few adverse effects of the drug, and developed no dependence on its consumption. The effect of meloxicam very slowly decreased after weaning, vanishing in a total of 51 hours. Meloxicam is thus safer than diclofenac, does not induce dependence and stays efficient during a long lasting time period. It should thus be preferred to diclofenac in case of inflammatory reaction. The entire work is related in. ${ }^{31}$

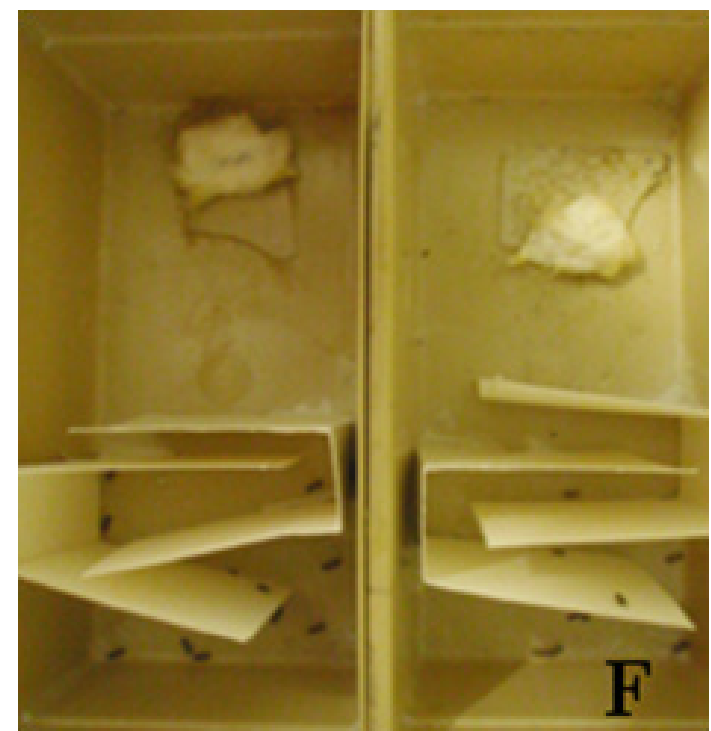

Figure IF Ants under diclofenac diet unable to cross a path with twists and turns, having thus their audacity and their cognition affected by the drug.

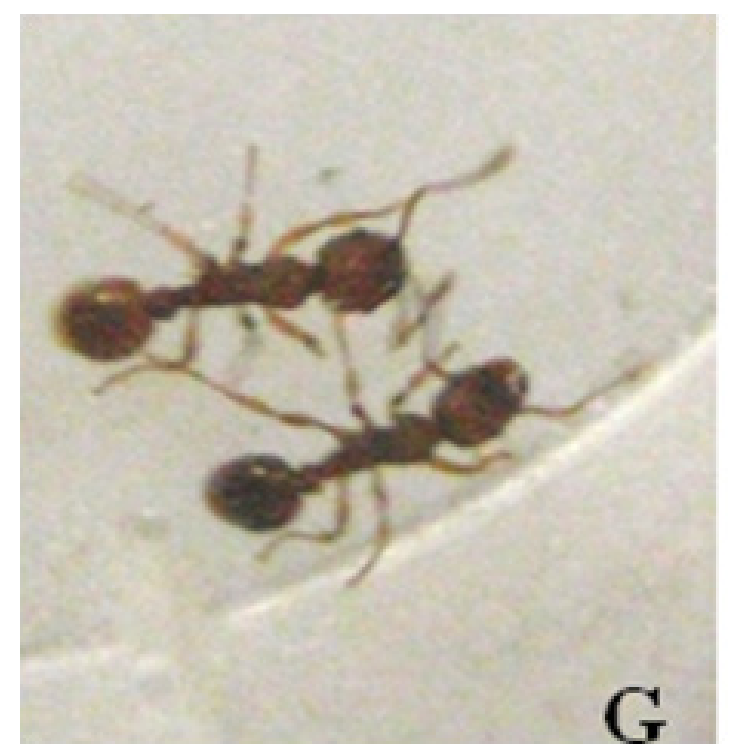

Figure IG Two ants under meloxicam diet staying side by side with no aggressiveness.

\section{Etoricoxib}

This anti-inflammatory drug affected the ants' food consumption, activity, locomotion, cognition, escaping capability, conditioning acquisition and memory. It increased the ants' tactile perception and aggressiveness against aliens (Figure 1H). Etoricoxib did not impact 
the ants' orientation ability, audacity, brood caring and aggressiveness against nestmates. The ants did not adapt themselves to the adverse effects of the drug, and developed no dependence on its consumption. After weaning, the effect of etoricoxib slowly decreased and vanished in 21-23hours (Figure 2). This anti-inflammatory drug is thus somewhat less safe than meloxicam, but safer than diclofenac and induce no dependence. Consequently, the best of the three examined anti-inflammatory drugs seems to be meloxicam, but research on the recent 'coxibs' is still in the process, and better drugs might be discovered. Details are in. ${ }^{32}$

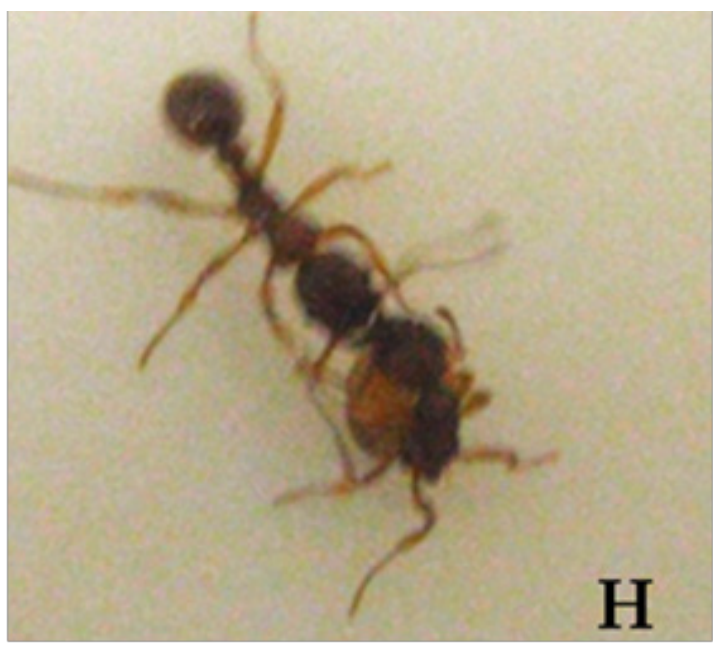

Figure I H An ant under etoricoxib diet soon attacking and stinging an alien.
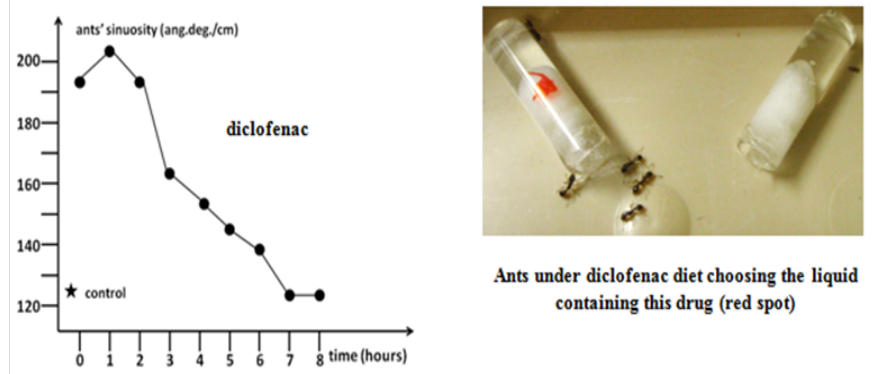

Ants under diclofenac diet choosing the liquid containing this drug (red spot)
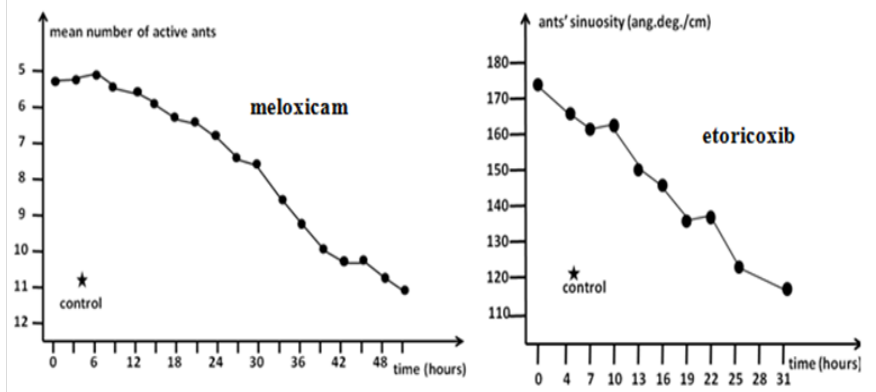

Figure 2 Decrease of the effect of three anti-inflammatory drugs after weaning. Diclofenac lost its effect in 6 hours, what leaded to dependence (photo). Meloxicam lost its effect in about $5 \mathrm{I}$ hours; it induced no dependence and had only few adverse effects: it is the best of the three examined antiinflammatory drugs. Etoricoxib lost its effect in 2I-23 hours; it induced no dependence, but had some adverse effects: it is thus less recommended than meloxicam.

\section{Conclusion}

On basis of the seven here briefly reported studies, the following use of the examined products could be advised. Green clay could be consumed in case of stomach hyperacidity, instead of the drugs commonly used for this purpose and which are not without adverse effects. As inferred from observations made on ants, the beneficial effect of glucosamine may be due to the fact that it increases movement and activity. Sildenafil appears to be not safe because it reduces the individual' perception (for instance the visual perception), this being due to its physiological functioning. It should be consumed only occasionally. Homeopathic drugs may be in some cases (state for stress of instance), under some dilutions, and for some patients, rather efficient without presenting adverse effects. Their use could be accepted by practitioners. Concerning the anti-inflammatory drugs, the nowadays safest one appears to be meloxicam. At a general point of view, ants appeared to be good biological models. Several colonies, each containing hundreds of workers, can be easily maintained in a laboratory, and many physiological and ethological traits can be examined on them. One of these traits is the dependence on a product, which occurs when the effect of the product quickly decreases after weaning. Also, the decrease of a product after weaning can be defined using ants as models, what allows defining the time at which the patients will need the product again. In fact, each new drug or product devoted to human or animal use should be firstly tested on biological models. The results of these studies should be clearly, objectively, and without conflict of interest reported, what is not always the case. We many times discovered that compromising studies had not been divulgated, but also found a lot of excellent studies made by honest researchers or practitioners. Medicinal staff is a world, with its cheaters and its heroes.

\section{Acknowledgments}

We are very grateful to Roger Cammaerts who meticulously corrected a first version of the present paper.

\section{Conflicts of interest}

We affirm having maintained the ants in the best possible conditions. We also affirm having no conflict of interest at all concerning the use of any studied drugs or products. We are ethologists, working on ants, and receiving no money for making our research.

\section{References}

1. Cammaerts MC, De Doncker P, Patris X, et al. GSM $900 \mathrm{MHz}$ radiations inhibits ants' association between food sites and encountered cues. Electrom Biol Med. 2012;31(2):151-165.

2. Sherwood L, Klandorf H, Yancey P. Physiologie animale. De Boeck supérieur, editor. 2016. 904 p.

3. Bousquet C. Bêtes de science. 2003. 240 p.

4. Andre RG, Wirtz RA, Das YT. Insect Models for Biomedical Research. In: Woodhead AD, editor. Non mammalian Animal Models for Biomedical Research. USA: Boca Raton, CRC Press; 1989.

5. Cammaerts MC, Cammaerts D. Comparative outlook over three $\mathrm{Myrmica}$ species' biotopes and foragers' know-how. Biologia. 2014;69(8):10511058.

6. Cammaerts MC, Cammaerts R. The Acquisition of Cognitive Abilities by Ants:A Study on Three Myrmica Species (Hymenoptera, Formicidae). Adv Stud Biol. 2015;7(7):335-348.

7. Cammaerts MC. Can Myrmica rubra ants use tools or learn to use them? Int J Biol. 2018;10 (1):1-12.

8. Cammaerts MC, Cammaerts R. Ants are at the first stage of the notion of zero. Int J Biol. 2019;11(1):54-65.

9. Cammaerts MC. Ants as biological models for studying effects of 
substances used by humans. JSM Anatomy and Physiology. 2016. 8 p.

10. Cammaerts MC. Some findings on ants as models, which should be considered for caring of humans. MOJ Biol Med. 2017;1(5):00027.

11. Cammaerts MC. Ants as models for examining potential adverse effects of products used by humans. JSM Anatomy and Physiology. 2018;3(1):1016.

12. Wandel S, Jüni $\mathrm{P}$, Tendal B, et al. Effects of glucosamine, chondroitin, or placebo in patients with osteoarthritis of hip or knee: network metaanalysis. BMJ .2010;341:c4675.

13. Pavelká K, Gatterová J, Olejarová M, et al. Glucosamine Sulfate Use and Delay of Progression of Knee Osteoarthritis. Arch Int Med. 2002;162(18):2113-2123.

14. Balkan B, Dunning BE. Glucosamine Inhibits Glucokinase In Vitro and Produces a Glucose-Specific Impairment of In Vivo Insulin Secretion in Rats. Diabetes. 1994;43(10):1173-1179.

15. Trent Medicines Information. Clinical evidence for homeopathy. Specialist Pharmacy Service. Meicester and Regional Drug \& Therapeutic Centre. Newcastel. 2017.

16. National Health and Medical Research Council. Frequently asked questions arising from public consultation: NHMRC Advice on the effectiveness of homeopathy for treating health conditions. National Health and Medical Research Council. 2015. 12 p.

17. Danno K, Colas A, Masson JL, et al. Homeopathic treatment of migraine in children: results of a prospective, multicenter, observational study. $J$ Altern Complement Med. 2013;10(2):119-123

18. Green RE, Taggart MA, Das D, et al. Collapse of Asian vulture populations: risk of mortality from residues of the veterinary drug diclofenac in carcasses of treated cattle. Journal of Applied Ecology. 2006;43(5):949-956.

19. Schmidt M. Sorensen HY, Pederson L. Diclofenac use and cardiovascular risks: series of nationwide cohort studies. British Medical Journal. 2018;362:k3426.

20. Valat JP, Accardo S, Reginster JY, et al. A comparison of the efficacy and tolerability of meloxicam and diclofenac in the treatment of patients with osteoarthritis of the lumbar spine. Inflammation Research. 2001;50(Suppl

\section{1):S30-S34}

21. Farkouh ME, Greenberg BP. An evidence-based review of the cardiovascular risks of non steroidal anti-inflammatory drugs. The American Journal of Cardiology. 2009;103(9):1227-1237.

22. Everts B, Wahborg P, Hedner T. COX-2-Specific Inhibitors - the Emergence of a New Class of Analgesic and Anti-inflammatory Drugs. Clinical Rheumatology. 2000;19(5):331-343.

23. Shi S, Klotz U. Clinical use and pharmacological properties of selective COX-2 inhibitors. European Journal of Clinical Pharmacology. 2008;64(3):233-252.

24. Cammaerts MC, Morel F, Martino F, et al. An easy and cheap softwarebased method to assess two-dimensional trajectory parameters. Belg $J$ Zool. 2012;142(2):145-151.

25. Cammaerts MC, Cammaerts R. Green clay used as a remedy for gastric hyperacidity has no harmful effect (a study on ants as models). Acto Scientific Pharmaceutical Sciences. 2018;2(7):38-44.

26. Cammaerts MC, Cammaerts R. Safety of glucosamine, examined on ants as models. MOJ Biology and Medicine. 2018;3(4):132-142.

27. Cammaerts MC, Cammaerts R. Is the largely used sildenafil citrate without harmful effect? A study on ants as models. EC Pharmacology and Toxicology. 2018;6(8):730-747.

28. Cammaerts MC, Cammaerts D. Challenging the safety and efficiency of homeopathy: Ignatia amara as an example, ants as models. MOJ Biology and Medicine. 2019;4(1):1-14.

29. Cammaerts MC. Physical dependence on a substance occurs when the effect of this substance rapidly decreases after withdrawal. JSM Anatomy and Physiology. 2018;3(1):1017.

30. Cammaerts MC, Cammaerts R. Adverse effects of diclofenac observed on ants as models. Acta Scientific Pharmaceutical Sciences. 2019;3(3):2-13.

31. Cammaerts MC, Cammaerts R. Safety of meloxicam compared to that of diclofenac using ants as models. Acta Scientific Pharmaceutical Sciences. 2019;3(4):45-58.

32. Cammaerts MC, Cammaerts R. Adverse effects of etoricoxib, a selectively inhibiting cyclo-oxygenase-2 anti-inflammatory drug, assessed on ants used as a model. EC Pharmacology and Toxicology. 2019;7(5):12. 